\title{
A Study on Prediction of Weld Geometry in Laser Overlap Welding of Low Carbon Galvanized Steel Using ANN-Based Models
}

\author{
Kamel Oussaid, Abderazak El Ouafi \\ Department of Engineering, University of Quebec at Rimouski, Rimouski, QC, Canada \\ Email: Abderrazak_elouafi@uqar.ca, Kamel.Oussaid@uqar.ca
}

How to cite this paper: Oussaid, K. and El Ouafi, A. (2019) A Study on Prediction of Weld Geometry in Laser Overlap Welding of Low Carbon Galvanized Steel Using ANN-Based Models. Journal of Software Engineering and Applications, 12, 509-523. https://doi.org/10.4236/jsea.2019.1212031

Received: November 15, 2019

Accepted: December 20, 2019

Published: December 23, 2019

Copyright (c) 2019 by author(s) and Scientific Research Publishing Inc. This work is licensed under the Creative Commons Attribution International License (CC BY 4.0).

http://creativecommons.org/licenses/by/4.0/

\begin{abstract}
Predictive modelling for quality analysis becomes one of the most critical requirements for a continuous improvement of reliability, efficiency and safety of laser welding process. Accurate and effective model to perform non-destructive quality estimation is an essential part of this assessment. This paper presents a structured approach developed to design an effective artificial neural network based model for predicting the weld bead dimensional characteristic in laser overlap welding of low carbon galvanized steel. The modelling approach is based on the analysis of direct and interaction effects of laser welding parameters such as laser power, welding speed, laser beam diameter and gap on weld bead dimensional characteristics such as depth of penetration, width at top surface and width at interface. The data used in this analysis was derived from structured experimental investigations according to Taguchi method and exhaustive FEM based 3D modelling and simulation efforts. Using a factorial design, different neural network based prediction models were developed, implemented and evaluated. The models were trained and tested using experimental data, supported with the data generated by the $3 \mathrm{D}$ simulation. Hold-out test and $\mathrm{k}$-fold cross validation combined to various statistical tools were used to evaluate the influence of the laser welding parameters on the performances of the models. The results demonstrated that the proposed approach resulted successfully in a consistent model providing accurate and reliable predictions of weld bead dimensional characteristics under variable welding conditions. The best model presents prediction errors lower than $7 \%$ for the three weld quality characteristics.
\end{abstract}

\section{Keywords}

Laser Welding, Overlap Welding Configuration, Low Carbon Galvanized Steel, Weld Geometry, Artificial Neural Network, Predictive Modelling 


\section{Introduction}

Laser welding is an assembly process widely used in the industry, including the automotive industry. Overlap welding of galvanized steels enables joining of body car elements from different thicknesses. The disadvantage of the overlap configuration is the premature vaporization of zinc, which generates pressure at the interface of the overlapped sheets. These pressurized vapors eventually eject the metal out of the melting pool or trapped as blowers after solidification.

Several experimental studies have shown the possibility of overcoming this situation by controlling the welding process parameters. Like the keyhole welding, which creates a channel permitting the evacuation of zinc vapors, an optimal gap between the parts to be welded also allows the lateral evacuation of these vapors. This means that a good control of welding parameters and conditions (laser power, welding speed, focal diameter, Gap between sheets and sheet thicknesses) can produce the desired welds characteristics.

Laser welding parameters play an important role in determining the mechanical characteristics of the weld seam [1]. Since the resistance of welded joints is linearly proportional to the shape and dimensions of the weld cross section, it is obvious to identify the relationship between the welding parameters and the geometrical attributes of the weld. To obtain welds with well-defined characteristics, the traditional test-failure method proves too expensive and more time-consuming. Finite element modeling has led to promising results through the performance of simulation software, but the complexity of the laser welding process, which includes many physical phenomena (thermal transfer, fluid flow, electromagnetic and thermodynamics), makes the models very complex; therefore, the calculation time becomes too long.

In contrast, thanks to their strong learning ability, artificial neural networks (ANNs) can establish nonlinear deterministic relationships between the inputs and the outputs of any system regardless of their complexity. ANNs are inspired by the human brain, they can learn and experience from examples, as they have a powerful ability to classify and recognize patterns. ANNs are used in many different fields of business, industry and science [2].

Synthia et al. [1] have developed a neural network-based prediction model of weld bead geometry according to laser welding parameters, in three different shielding gases (argon, helium and nitrogen). Another study applied an artificial neural network to predict a geometry of GMAW welds with alternating shielding gases. The experimental results proved conformity and accordance with the predicted geometries. Furthermore, a sensitivity analysis showed that the welding speed is the most influential input parameter in the predicting model [3].

Frason et al. [4] developed a laser weld penetration monitoring system using an ANN to analyze acoustic emissions from the weld, and thereby determine the depth of penetration of the weld in real time. The system uses a fuzzy logic algorithm to compute the necessary speed changes to maintain the penetration at desired levels. An identical study used an ANN model to diagnose welding 
faults. Features extracted from the acoustic signals were used to train the ANN. After training, the ANN could identify normal and abnormal welds [5]. In the same vein, an ANN model uses data extracted from infrared thermal images that are recorded in real time during A-TIG welding, for the purpose of weld width and depth estimation [6]. Another study shows the possibility of using neural networks in the ascertainment of the weld quality for thermoplastics welded by laser transmission [7].

Olabi et al. [8] combined a backpropagation ANN with Taguchi design for experiments in order to optimize the parameters level (welding speed, laser power and focal position) of $\mathrm{CO} 2$ keyhole laser welding process in butt joint configurations. The laser welding of stainless steel in butt joint configuration was modeled using backpropagation trained neural network. The predicted weld dimensions showed conformance with the measured weld cross sections [9]. Castiliano et al. integrated an artificial neural network into the laser welding process, in order to optimize process parameters when welding stainless steel and increase its efficiency [10].

Depending on their architecture and their fields of application, several types of ANN exist. A broader description of different neural networks is presented in the literature review made by Zhang et al. [2]. The Multi-layer feedforward ANN is the most popular and widely used ANN in many applications, especially forecasting, because of their great ability to map nonlinear and complex relationships in multi-inputs multi-output context [11] [12] [13]. Jacques et al. proposed an ANN based predictive modelling approach for weld shape and dimensions in butt joint laser welding of galvanized steel [14]. The resulting model presents excellent predictions with an average error less than $10 \%$. This model, however, can only be applied in the case of laser welding in butt joint configurations.

Only a few studies used ANNs to predict the quality of laser welding of galvanized steels and even less in overlap configurations. The few attempts revealed in the literature are focussed on specific application of the ANNs without explicit and detailed references to the nature of the data used in the models training and validation and criteria adopted for the model performance evaluation. These fundamental ideas that constitute the basic ingredients of any models optimization procedure are indispensable to build an efficient predictive modeling approach.

The present paper presents an artificial neural network based model for predicting the weld bead dimensional characteristic in laser overlap welding of low carbon galvanized steel. The modelling approach is based on laser welding parameters such as laser power, welding speed, laser beam diameter and the gap between the overlapped parts to estimate specific weld bead dimensional characteristics such as depth of penetration, width at top surface and width at interface. A series of data provided from experiments using a $3 \mathrm{KW}$ Nd-YAG laser source in a well-structured Taguchi design are combined with simulation data provided by a 3D finite element model to train and test the ANN built using variables selection based factorial design. Hold-out test and k-fold cross validation 
combined to various improved statistical criteria are used for assessing the models performance.

\section{Modeling Approach}

\subsection{Methodology}

The purpose of this study is to set up a model able to predict accurately and quickly three geometrical characteristics of the weld seams: depth of penetration (DOP), weld width at the top surface (WS) and at the interface (WI) as defined in Figure 1. The three welds characteristics prediction in overlap laser welding configuration of low-carbon galvanized steel is based on laser power $(\mathrm{P})$, welding speed (S), laser beam diameter (D) and Gap (Gap) between the overlapped sheets as variables. The prediction model is achieved using an artificial neural network-based model (ANN).

In order to know the most influential laser welding parameters on the model accuracy, as well as the effects of these parameters on the quality of the of weld characteristics prediction, 16 models are built according to a full factorial design including the variables known for their influence on the variation of the geometrical characteristics of the weld. As shown in Table 1, a variable takes the value 1 when it is included in the model and 0 when it is not.

To eliminate the maximum random error sources, the learning data inputs, and the testing data inputs must represent the same population, i.e. both should be contained in the same variation range. This is the case in the present study, as shown in Table 2. Three levels are assigned to each parameter. The upper and lower limits of factors are respectively 2000 to $3000 \mathrm{~W}$ for the power of the laser, 40 to $70 \mathrm{~mm} / \mathrm{s}$ for the welding speed, 300 to $490 \mu \mathrm{m}$ for the beam diameter and 0.05 to $0.15 \mathrm{~mm}$ for the Gap. The Data assigned for training and testing the various models are partly provided from an experimental investigation of laser welding process [15] while the other part is produced by a 3D FEM simulation [16]. In order to include the gap in the ANN modeling, the finite element model is adapted for each Gap value $(0.05,0.1$ and $0.15 \mathrm{~mm})$ by recalculating the calibration coefficients ( $m$ and $n$ ) of the heat source for each time step.

The database generated by simulations is structured in a full factorial design of 4 factors, each at three levels, while experimental data are planned in three L9

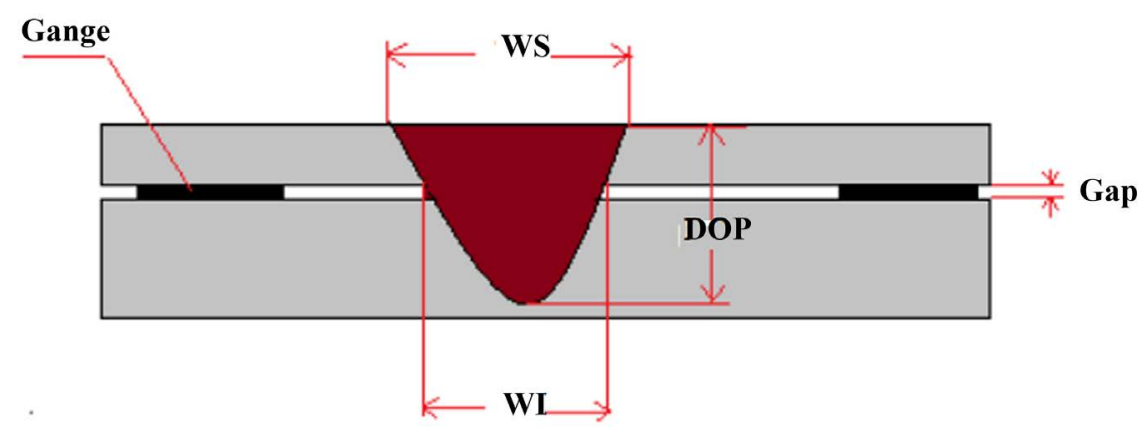

Figure 1. Geometric characteristics of weld cross section in overlap configuration. 
Table 1. Input variables of each of 16 ANN models.

\begin{tabular}{ccccc}
\hline Model & Gap & Power & Speed & Diameter \\
\hline $\mathrm{M}_{1}$ & 1 & 1 & 1 & 1 \\
$\mathrm{M}_{2}$ & 0 & 1 & 1 & 1 \\
$\mathrm{M}_{3}$ & 1 & 0 & 1 & 1 \\
$\mathrm{M}_{4}$ & 1 & 1 & 0 & 1 \\
$\mathrm{M}_{5}$ & 1 & 1 & 1 & 0 \\
$\mathrm{M}_{6}$ & 0 & 0 & 1 & 1 \\
$\mathrm{M}_{7}$ & 0 & 1 & 0 & 1 \\
$\mathrm{M}_{8}$ & 0 & 1 & 1 & 0 \\
$\mathrm{M}_{9}$ & 1 & 0 & 0 & 1 \\
$\mathrm{M}_{10}$ & 1 & 0 & 1 & 0 \\
$\mathrm{M}_{11}$ & 1 & 1 & 0 & 0 \\
$\mathrm{M}_{12}$ & 1 & 0 & 0 & 0 \\
$\mathrm{M}_{13}$ & 0 & 1 & 0 & 0 \\
$\mathrm{M}_{14}$ & 0 & 0 & 0 & 0 \\
$\mathrm{M}_{15}$ & 0 & 0 & 0 & 1 \\
$\mathrm{M}_{16}$ & 0 & 0 & 0 \\
\hline & & 0 & 0 \\
\hline
\end{tabular}

Table 2. Levels of process parameters.

\begin{tabular}{ccccc}
\hline Level & Gap & Power & Speed & Diameter \\
\hline 1 & 0.05 & 2000 & 40 & 300 \\
2 & 0.10 & 2500 & 55 & 395 \\
3 & 0.15 & 3000 & 70 & 490 \\
\hline
\end{tabular}

orthogonal matrix. For each gap value, to determine the true prediction errors of an ANN model and its accuracy for future predictions, new unused data in learning stage should be used in model testing phase, because learning errors are often inferior to validation errors. To do this, two validation methods are adopted: "hold-out set method" and " $k$-fold crossvalidation method". The hold-out set method consists of using the large part of the data to train the model and the remaining data to test it. The $k$-fold cross validation method consists of sampling all the $\mathrm{n}$ data after its randomization in $\mathrm{k}$ segments, the model is then trained by $n-k$ data and tested by $k$ remaining data. The procedure is repeated $k$ times by changing the testing sample each time. The validation errors are estimated by various statistical tools for the $k$ variants, then averaged to determine the real prediction errors of the model.

The neural network modeling procedure used in this study consists first in confirming the reliability of the data provided by the $3 \mathrm{D}$ finite element model. Using the hold-out set method, the ANN models are trained by the entire simu- 
lation data and then tested by the experimental data. Second, all the data are mixed and randomized, then the $k$-fold cross validation method is applied, with $k=6$.

\subsection{ANN Model Building}

There are several kinds of networks according to their architectures, their internal mechanisms and their application objectives. In the present study, the interest is focused on multilayer feedforward back propagation perceptron for its prediction capability. As illustrated in Figure 2, the MLP is mainly composed of: 1) an input layer with a number of neurons equals the number of input variables of the ANN, called independent variables. 2) One or more hidden layers having a limited number of neurons $(n)$. It is necessary to try several networks for different values of $\mathrm{n}$ in order to optimize the training performances especially as the number of variables varies from one model to another and to avoid overtraining. Consequently, to avoid long training and overfitting that could affect the models accuracy, only one hidden layer is considered using $n=2 \times p+1$, with $p$ is the number of independent variables. 3) An output layer containing a specific number of neurons equal to the number of output variables. In our case, there are three outputs (DOP, WS and WI).

The artificial neuron is an integrator that performs the weighted sum of its inputs originate from the previous layer (Equation (1)). The resulted sum is then transformed by a transfer function to provide the neuron output. The transfer function used for neurons of the input and hidden layer, is a sigmoid (Equation
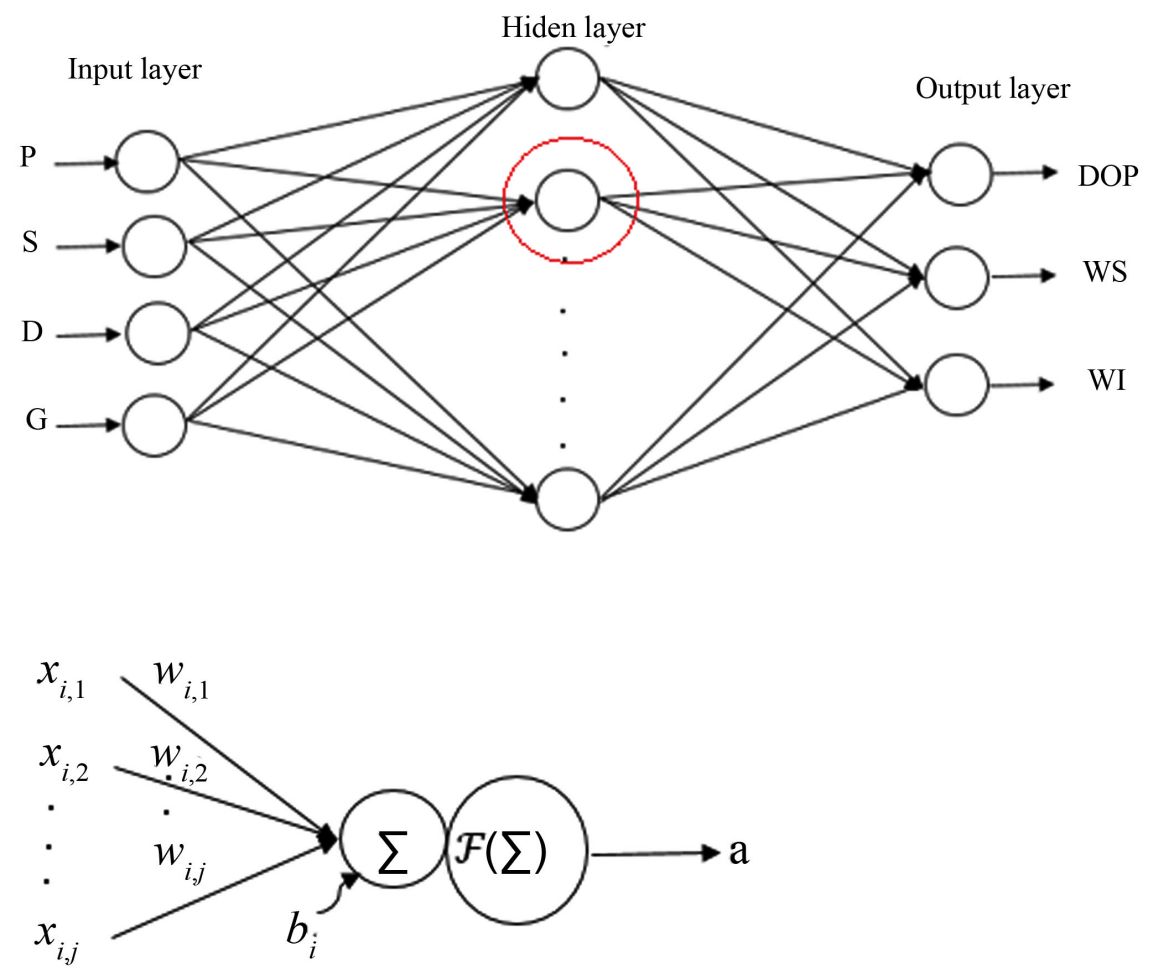

Figure 2. Neural network architecture. 
(2)), and that used for the output layer neurons is a linear function (Equation (3)) where $x, w^{T}$ and $b$ denote respectively neuron input, Wight matrix and bias.

$$
\begin{gathered}
a=f(s)=f\left(x W^{T}-b\right) \\
f(s)=1 /\left(1+e^{-s}\right) \\
f(s)=s
\end{gathered}
$$

The outputs of last layer neurons are then compared to the target values (dependent variables), if the difference is greater than the tolerated deviation, the network updates the weights associated to each neuron by means of a backpropagation technique and therefore starts a new computing loop, to help minimize the gap between the network output and the target value. Thus, the iterations continue until reaching a tolerated value of the error. The networks are assembled using the built in Matlab NetfitingToolbox. The function used for the training of the various networks is the Levenberg-Marquardt function. This function use an algorithm for supervised learning considered the fastest available algorithm by virtue of the validation vectors, which enables the network learning to stop prematurely if the performance in the validation matrix fails to reduce the error.

\subsection{Model Assessment}

Based on the modeling process results, three statistical variables are estimated to evaluate the performance of each model: 1) Coefficient of determination $R^{2}$ which is commonly applied to training errors. Its main defect is its growth with the addition of input variables to the model, whereas an excess of variables does not always lead to robust models. This is why one is interested in the adjusted coefficient $\overline{R^{2}}$. 2) Root mean squared error (RMSE) is the standard deviation of prediction errors (residuals), it measures the extent of these residuals and indicates the concentration of data around the line of best fit, and 3) mean absolute percentage error (MAPE) is a useful measure of forecasting accuracy. It is easy to interpret because it is expressed in percentage. The criteria are expressed mathematically as:

$$
\begin{aligned}
R^{2} & =1-\frac{\sum_{i=1}^{n}\left(y_{i}-\hat{y}_{i}\right)^{2}}{\sum_{i=1}^{n}\left(y_{i}-\bar{y}\right)^{2}} \\
\overline{R^{2}} & =1-\frac{\left(1-R^{2}\right)(n-1)}{(n-p-1)} \\
\text { MAPE } & =\left(\frac{1}{n} \sum_{i=1}^{n}\left|\frac{y_{i}-\hat{y}_{i}}{y_{i}}\right|\right) 100(\%) \\
\text { RMSE } & =\frac{1}{n} \sqrt{\sum_{i=1}^{n}\left(y_{i}-\hat{y}_{i}\right)^{2}}
\end{aligned}
$$

where $n, p, y_{i}, \hat{y}$ and $\bar{y}$ denote respectively sample size, number of input process parameter, actual output, predicted output and the mean actual output. 


\section{Results and Discussion}

The evaluation of the training and testing performances of the 16 models are based on the three statistical criteria applied to the two validation methods. First, the models training and testing performances using the hold-out set method are evaluated and the contributions of the laser welding parameters to ANN model improvement are estimated. In this case, as the models are trained using numerical simulation data and tested by means of experimental data, the major part of the training errors are due to the laser welding parameters not considered as variables in the ANN model building and to the possible bias in the 3D numerical model predictions, while the observed testing errors are related to experimental errors.

Table 3 illustrates the learning error estimates of the 16 ANN models using the three performance indicators mentioned above, namely RMSE, MAPE and $\overline{R^{2}}$. The comparison between the learning performances of the 16 models shows the contribution of the various welding parameters to the improvement of the model performances. The results show that the best model for the prediction of the three weld seam attributes (DOP, WS and WI) is indeed the model $M_{1}$, which contains the four process variables. The relative errors of DOP, WS and WI Prediction are $2.1 \%, 1.1 \%$ and $2.6 \%$ respectively. The $\mathrm{M}_{2}$ model, which does not include the gap as input, also shows high performances in the prediction of

Table 3. Training performance of the models using hold-out set method.

\begin{tabular}{|c|c|c|c|c|c|c|c|c|c|}
\hline \multirow{2}{*}{ Model } & \multicolumn{3}{|c|}{ DOP } & \multicolumn{3}{|c|}{ WS } & \multicolumn{3}{|c|}{ WI } \\
\hline & RMSE & MAPE & $\overline{R^{2}}$ & RMSE & MAPE & $\overline{R^{2}}$ & RMSE & MAPE & $\overline{R^{2}}$ \\
\hline $\mathrm{M}_{1}$ & 68.9 & 2.1 & 1 & 20.2 & 1.1 & 1.01 & 53.5 & 2.6 & 0.99 \\
\hline $\mathrm{M}_{2}$ & 142.3 & 4.2 & 0.95 & 35.5 & 2 & 0.97 & 80 & 4.6 & 0.89 \\
\hline $\mathrm{M}_{3}$ & 263 & 9.3 & 0.79 & 119.3 & 7.1 & 0.50 & 179 & 10.4 & 0.53 \\
\hline $\mathrm{M}_{4}$ & 440.4 & 16.4 & 0.29 & 111.1 & 4.1 & 0.58 & 193 & 10.8 & 30.9 \\
\hline $\mathrm{M}_{5}$ & 237.9 & 8.2 & 0.82 & 47.8 & 2.8 & 0.93 & 79.8 & 4.5 & 0.89 \\
\hline $\mathrm{M}_{6}$ & 281.5 & 9.7 & 0.74 & 124 & 7.4 & 0.48 & 191 & 11.2 & 0.48 \\
\hline $\mathrm{M}_{7}$ & 449.2 & 16.2 & 0.27 & 107.6 & 6.2 & 50.8 & 196.8 & 11.4 & 0.36 \\
\hline $\mathrm{M}_{8}$ & 268.9 & 8.9 & 0.77 & 57.5 & 3.2 & 0.89 & 105.3 & 6.1 & 0.83 \\
\hline $\mathrm{M}_{9}$ & 476.2 & 16.9 & 0.16 & 147.6 & 8.4 & 0.17 & 238.9 & 13.3 & 0.064 \\
\hline $\mathrm{M}_{10}$ & 330.1 & 11.1 & 0.64 & 130.4 & 7.8 & 0.36 & 184.2 & 11 & 0.48 \\
\hline $\mathrm{M}_{11}$ & 476.7 & 17.0 & 0.16 & 117.7 & 6.8 & 0.50 & 191.3 & 11 & 0.375 \\
\hline $\mathrm{M}_{12}$ & 507.6 & 18.1 & 0.03 & 161 & 9.1 & 0.02 & 242.3 & 13.2 & 0.047 \\
\hline $\mathrm{M}_{13}$ & 487.1 & 17.2 & 0.12 & 121.8 & 7 & 0.47 & 204.6 & 11.8 & 0.32 \\
\hline $\mathrm{M}_{14}$ & 347.8 & 1.8 & 0.59 & 137.1 & 8.1 & 0.33 & 199 & 11.9 & 0.43 \\
\hline $\mathrm{M}_{15}$ & 486.1 & 17 & 0.12 & 151.7 & 8.5 & 0.15 & 250.9 & 14 & 0.018 \\
\hline $\mathrm{M}_{16}$ & 517.3 & 18.3 & 0 & 165.6 & 9.3 & 0 & 253.8 & 14.2 & 0 \\
\hline
\end{tabular}


the three weld seam attributes with respectively a relative error of $4.2 \%, 2 \%$ and 4.6\%. In third place comes the $\mathrm{M}_{5}$ model, which does not consider the focal diameter as input with prediction errors of $8.2 \%, 2.8 \%$ and $4.5 \%$ for DOP, WS and WI respectively. Among the two-variable models, the model $\mathrm{M}_{8}$, which considers only the power and the welding speed as inputs, shows relatively good performance during learning stage. The DOP, WS and WI relative prediction errors are $8.9 \%, 3.2 \%$ and $6.1 \%$ respectively.

Variance analysis (ANOVA) results in Table 4 and graphs of main effects presented in Figures 3-5 are the two statistical tools used to evaluate the contribution of the laser parameters, as well as their interactions in the reduction of the prediction error estimator (RMSE) for the three geometric welding attributes (DOP, WS and WI).

Despite their percentage differences, the graphs of effect reveal that all welding parameters have a positive effect on improving the prediction quality of the three geometric attributes of the weld. This asserts that the most accurate and reliable model is indeed the $\mathrm{M}_{1}$ which considers all the variables.

The $P$-value and $F$-value express the reliability of ANOVA results. For example, for $P=0.03$, the Gap contribution of $1.2 \%$ is a reliable result with $97 \%$ confidence. As we can see, the confidence interval for the effects of laser power, welding speed and focal diameter is about $99 \%$, and that associated with the gap effect is $94 \%$, this is most likely due to difficulty maintaining a constant Gap along the welding line.

A comparison between validation errors and learning errors shows how well an ANN model can predict the geometric attributes of the weld for any laser welding parameters combination. The comparison is applied to the models that show better performances during the learning process, namely models $M_{1}, M_{2}$, $\mathrm{M}_{5}$ and $\mathrm{M}_{8}$. Table 5 includes learning errors, validation errors, and $k$-fold crossvalidation error.

Table 4. Contribution of laser welding parameters to ANN model learning improvement.

\begin{tabular}{cccccccccc}
\hline \multirow{2}{*}{ Source } & \multicolumn{3}{c}{ RMSE_DOP } & \multicolumn{3}{c}{ RMSE_WS } & \multicolumn{3}{c}{ RMSE_WI } \\
\cline { 2 - 9 } & C\% & $F$-Value & $P$-Value & C\% & $F$-Value & $P$-Value & C\% & $F$-Value & $P$-Value \\
\hline Gap & 0.51 & 5.18 & 0.057 & 0.29 & 5.22 & 0.06 & 1.18 & 20.46 & 0.003 \\
$\mathrm{P}$ & 8.28 & 84.63 & 0.000 & 54.4 & 988.6 & 0.00 & 41 & 711.54 & 0.000 \\
$\mathrm{~S}$ & 77.5 & 792.4 & 0.000 & 34.4 & 624.5 & 0.00 & 50.1 & 868.69 & 0.000 \\
$\mathrm{D}$ & 6.42 & 65.6 & 0.000 & 2.70 & 48.9 & 0.00 & 0.43 & 7.43 & 0.030 \\
$\mathrm{P} * \mathrm{~S}$ & 3.25 & 3.25 & 0.001 & 7.34 & 133.3 & 0.00 & 6.18 & 107.2 & 0.000 \\
$\mathrm{P} * \mathrm{D}$ & 0.72 & 7.34 & 0.030 & 0.10 & 1.89 & 0.21 & 0.18 & 3.21 & 0.116 \\
$\mathrm{~S} * \mathrm{D}$ & 2.05 & 20.98 & 0.003 & 0.17 & 3.08 & 0.12 & 0.34 & 5.84 & 0.046 \\
$\mathrm{P} * \mathrm{~S}{ }^{*} \mathrm{D}$ & 0.53 & 5.42 & 0.053 & 0.23 & 4.2 & 0.08 & 0.17 & 3 & 0.127 \\
Error & 0.69 & - & - & 0.39 & - & - & 0.40 & - & - \\
Total & 100 & - & - & 100 & - & - & 100 & - & - \\
\hline
\end{tabular}




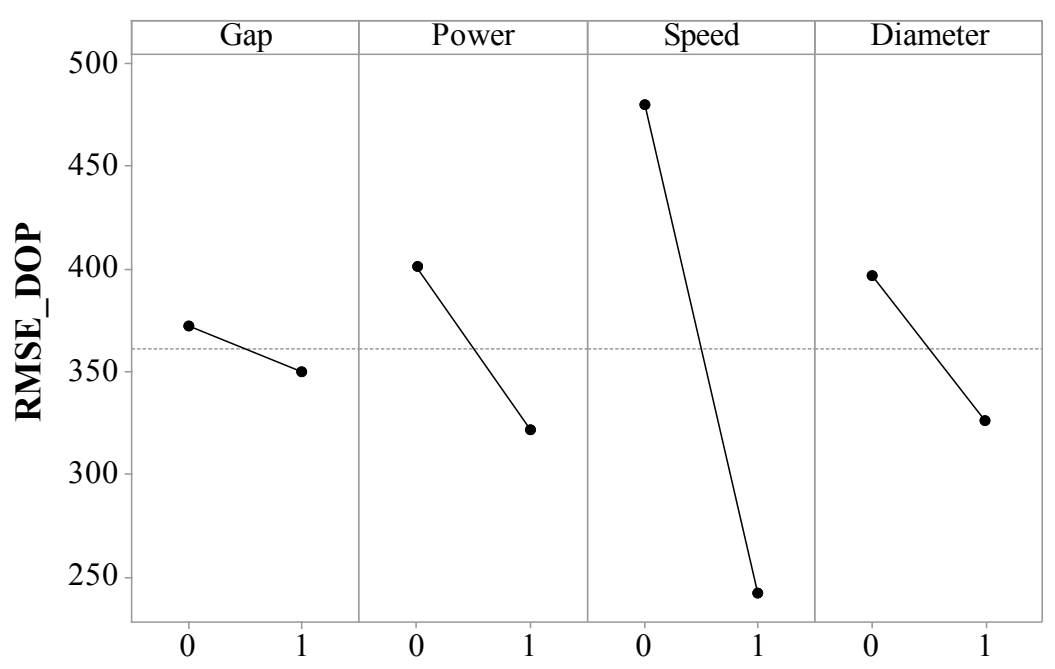

Figure 3. Laser welding parameters effects on training MSE_DOP reduction.



Figure 4. Laser welding parameters effects on training MSE_WS reduction.

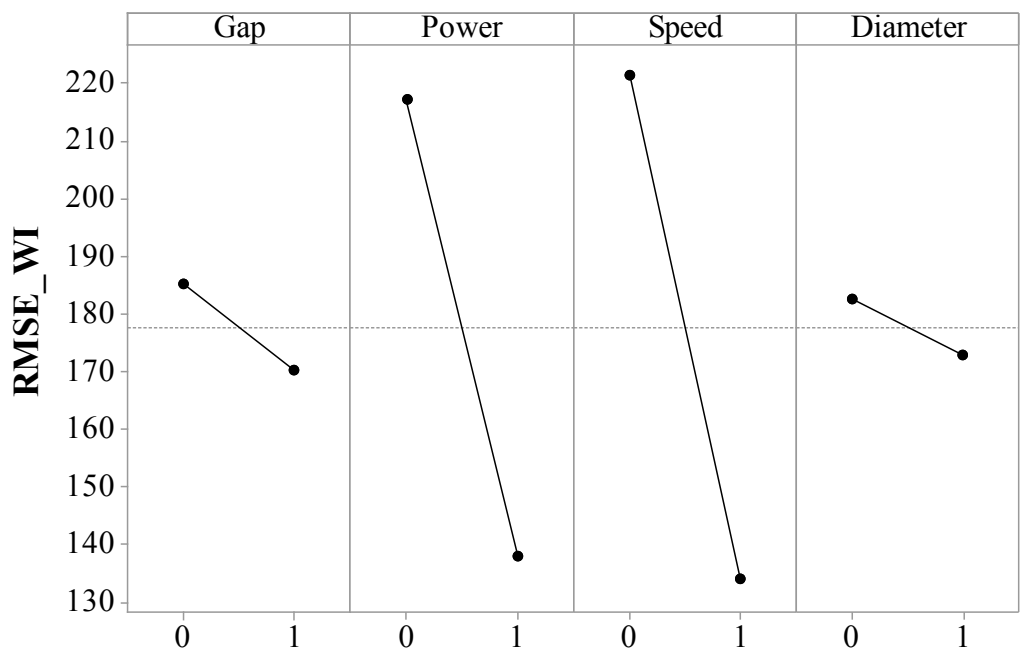

Figure 5. Laser welding parameters effects on training RMSE_WS reduction. 
Table 5. Error estimates of training, hold-out set validation $\left(\mathrm{Val}_{1}\right)$ and 6-fold cross validation $\left(\mathrm{Val}_{2}\right)$ of the best models.

\begin{tabular}{cccccccc}
\hline Model & Limits & RMSE_DOP & MAPE_DOP & RMSE_WS & MAPE_WS & RMSE_WI & MAPE_WI \\
\hline & Train & 68.9 & 2.1 & 20.2 & 1.1 & 53.5 & 2.6 \\
$\mathrm{M}_{1}$ & $\mathrm{Val}_{1}$ & 134.01 & 6.05 & 48.51 & 3.1 & 133.4 & 6.81 \\
& $\mathrm{Val}_{2}$ & 163.1 & 6.8 & 35.2 & 2.2 & 101.5 & 6.6 \\
& $\mathrm{Train}$ & 142.3 & 4.2 & 35.5 & 2.8 & 79.8 & 4.5 \\
$\mathrm{M}_{2}$ & $\mathrm{Val}_{1}$ & 194.6 & 8.2 & 62.4 & 4.2 & 166.4 & 8.7 \\
& $\mathrm{Val}_{2}$ & 203.3 & 8.8 & 50.5 & 3.4 & 117.4 & 8.4 \\
& $\mathrm{Train}$ & 237.9 & 8.2 & 47.8 & 2.8 & 79.8 & 4.5 \\
$\mathrm{M}_{5}$ & $\mathrm{Val}_{1}$ & 330.2 & 12.9 & 68.7 & 4.2 & 152.3 & 7.6 \\
& $\mathrm{Val}_{2}$ & 366.4 & 17.4 & 65.2 & 4.8 & 115.4 & 7.8 \\
& $\mathrm{Train}$ & 268.9 & 8.9 & 57.5 & 3.2 & 105.4 & 6.1 \\
$\mathrm{M}_{8}$ & $\mathrm{Val}_{1}$ & 337.2 & 13.6 & 80.5 & 4.9 & 168.4 & 8.7 \\
& $\mathrm{Val}_{2}$ & 308.5 & 13.0 & 66.8 & 4.4 & 126.6 & 8.5 \\
\hline
\end{tabular}

The results do not show a large deviation between training errors and validation errors, as the maximum gap between these two has been proved to not exceed $4 \%$. The validation errors obtained by the two methods are almost identical. In the light of the results shown in Table 5, the DOP can be predicted with a precision greater than $93 \%$ by the model $\mathrm{M}_{1}$, a precision greater than $91 \%$ by the $\mathrm{M}_{2}$ model and a prediction error exceeding $10 \%$ for the two other models. WS can be estimated with a precision greater than $95 \%$ by the four models. WI can be predicted by the model $M_{1}$ with an accuracy greater than $93 \%$ and a precision of $91 \%$ for other models. Figure 6 shows the values predicted by the model $M_{1}$ Vs actual values, respectively of DOP, WS and WI. Figure 7 shows those predicted by the model $M_{2}$ and Figure 8 shows those predicted by model $M_{5}$. The contour of the cross section of a weld bead can be deduced from the three predicted geometric attributes DOP, WS and WI.

\section{Conclusion}

This paper presents a structured approach developed to design an effective artificial neural network based model for predicting the weld bead dimensional characteristic in laser overlap welding of low carbon galvanized steel. Based on a fused data provided by structured experimental investigations using Taguchi method and in-depth FEM based 3D simulations, the possible relationships between welding parameters such as laser power, welding speed, laser beam diameter and gap, and weld bead dimensional characteristics such as depth of penetration, width at top surface and width at interface are analyzed and their sensitivity to the welding conditions are evaluated using relevant statistical tools. Based on these results, a factorial design is used to develop, implement and evaluate different neural network based prediction models. The proposed models 

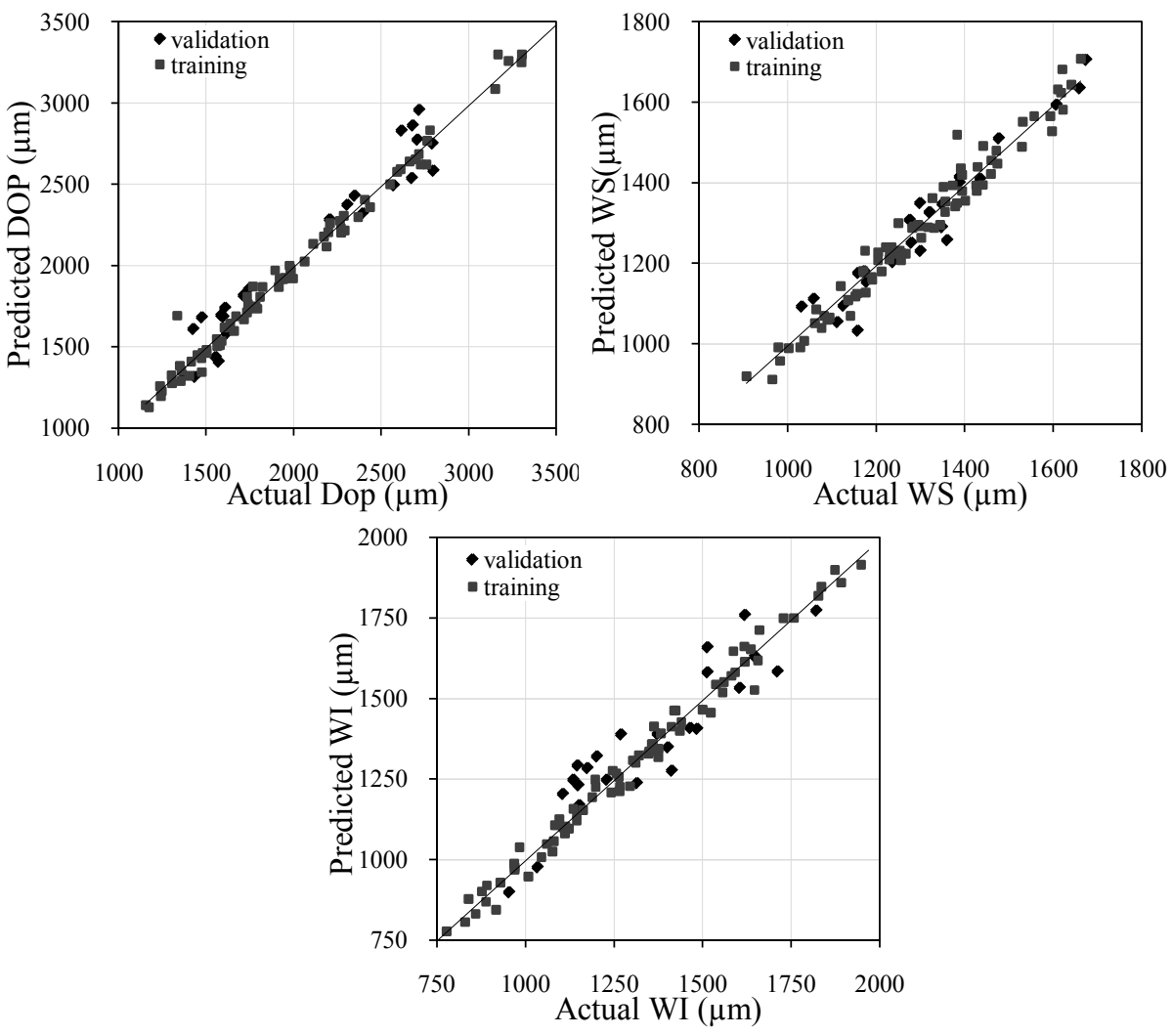

Figure 6. Predicted Vs actual DOP, WS and WI using $M_{1}$.
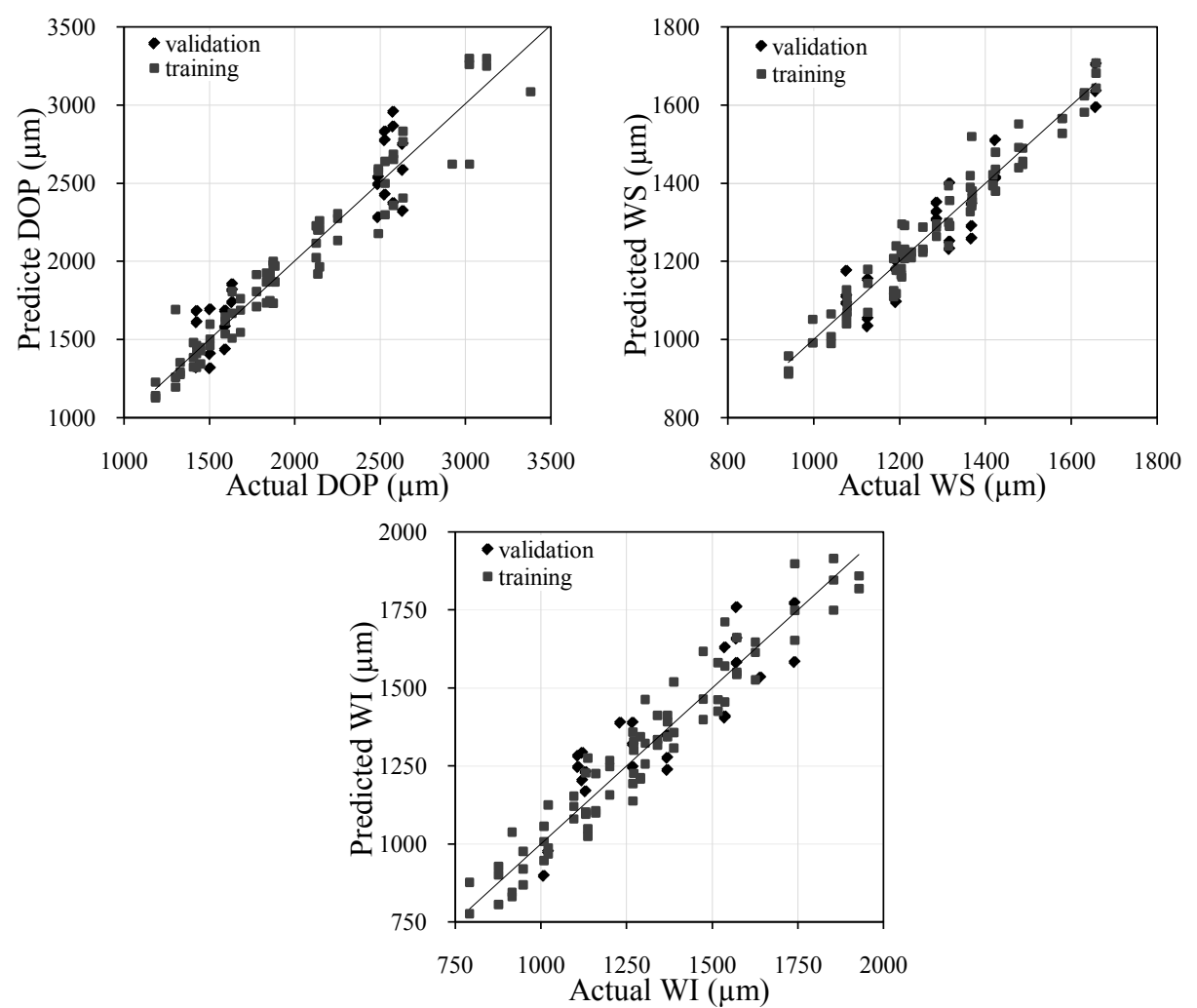

Figure 7. Predicted Vs actual DOP, WS and WI using $\mathrm{M}_{2}$. 

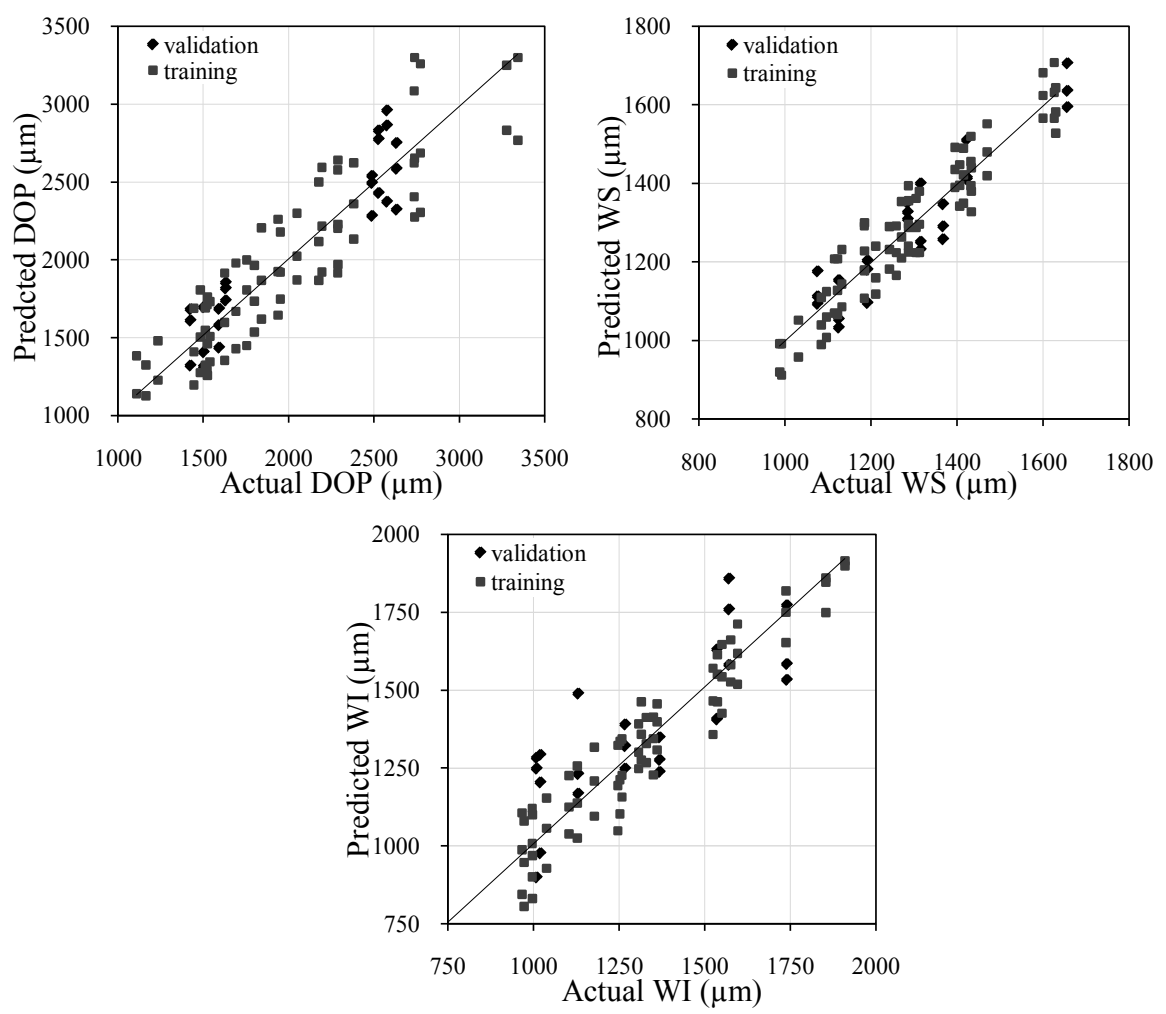

Figure 8. Predicted Vs actual DOP, WS and WI using $\mathrm{M}_{5}$.

are trained and tested using experimental data, supported by the data generated by the 3D simulation. Hold-out test and $k$-fold cross validation combined to improved statistical criteria are used to evaluate the influence of the laser welding parameters on the performances of the models. Analyses of variance results reveal that all the welding parameters have a positive contribution to the improvement of the prediction quality. The laser power and the welding speed contributions are much more important compared to the contribution of the laser beam diameter. The gap contribution appears to be insignificant.

The achieved predictive modelling results demonstrate that the resulting models present excellent performances and can effectively predict the weld bead dimensional characteristics with average predicting errors less than $10 \%$. The validation process reveals that the WS can be predicted with an accuracy of $96 \%$ while the prediction accuracy of DOP and WI is about $93 \%$. These results demonstrate that the proposed ANN based prediction approach can effectively lead to a consistent model able to accurately and reliably provide an appropriate prediction of weld bead dimensional characteristics in laser overlap welding of low carbon galvanized steel under variable welding parameters and conditions.

With the encouraging results achieved using this modelling strategy, the laser overlap welding of low carbon galvanized steel will be the subject of additional and exhaustive investigations to produce more numerical simulation and experimental data as well as to test others neural networks approach in order to develop more efficient ANN predictive modelling method. 


\section{Conflicts of Interest}

The authors declare no conflicts of interest regarding the publication of this paper.

\section{References}

[1] Sathiya, P., Panneerselvam, K. and Jaleel, M.A. (2012) Optimization of Laser Welding Process Parameters for Super Austenitic Stainless Steel Using Artificial Neural Networks and Genetic Algorithm. Materials \& Design, 36, 490-498. https://doi.org/10.1016/j.matdes.2011.11.028

[2] Zhang, G., Patuwo, B.E. and Hu, M.Y. (1998) Forecasting with Artificial Neural Networks: The State of the Art. International Journal of Forecasting, 14, 35-62. https://doi.org/10.1016/S0169-2070(97)00044-7

[3] Campbell, S., Galloway, A. and McPherson, N. (2012) Artificial Neural Network Prediction of Weld Geometry Performed Using GMAW with Alternating Shielding Gases. Welding Journal, 91, 174-181.

[4] Farson, D.F., Fang, K.S. and Kern, J. (1991) Intelligent Laser Welding Control. International Congress on Applications of Lasers \& Electro-Optics, Vol. 2, 104-112. https://doi.org/10.2351/1.5058430

[5] Luo, H., et al. (2005) Application of Artificial Neural Network in Laser Welding Defect Diagnosis. Journal of Materials Processing Technology, 170, 403-411. https://doi.org/10.1016/j.jmatprotec.2005.06.008

[6] Chandrasekhar, N., Vasudevan, M., Bhaduri, A.K. and Jayakumar, T. (2015) Intelligent Modeling for Estimating Weld Bead Width and Depth of Penetration from Infrared Thermal Images of the Weld Pool. Journal of Intelligent Manufacturing, 26, 59-71 https://doi.org/10.1007/s10845-013-0762-X

[7] Acherjee, B., Mondal, S., Tudu, B. and Misra, D. (2011) Application of Artificial Neural Network for Predicting Weld Quality in Laser Transmission Welding of Thermoplastics. Applied Soft Computing, 11, 2548-2555. https://doi.org/10.1016/j.asoc.2010.10.005

[8] Olabi, A.G., Casalino, G., Benyounis, K.Y. and Hashmi, M.S.J. (2006) An ANN and Taguchi Algorithms Integrated Approach to the Optimization of $\mathrm{CO}_{2}$ Laser Welding. Advances in Engineering Software, 37, 643-648.

https://doi.org/10.1016/j.advengsoft.2006.02.002

[9] Balasubramanian, K.R., Buvanashekaran, G. and Sankaranarayanasamy, K. (2010) Modeling of Laser Beam Welding of Stainless Steel Sheet Butt Joint Using Neural Networks. CIRP Journal of Manufacturing Science and Technology, 3, 80-84. https://doi.org/10.1016/j.cirpj.2010.07.001

[10] Casalino, G. and Minutolo, F.M.C. (2004) A Model for Evaluation of Laser Welding Efficiency and Quality Using an Artificial Neural Network and Fuzzy Logic. Journal of Engineering Manufacture, 218, 641-646. https://doi.org/10.1243/0954405041167112

[11] Meireles, M.R., Almeida, P.E. and Simões, M.G. (2003) A Comprehensive Review for Industrial Applicability of Artificial Neural Networks. IEEE Transactions on Industrial Electronics, 50, 585-601. https://doi.org/10.1109/TIE.2003.812470

[12] Paliwal, M. and Kumar, U.A. (2009) Neural Networks and Statistical Techniques: A Review of Applications. Expert Systems with Applications, 36, 2-17. https://doi.org/10.1016/j.eswa.2007.10.005

[13] Dagli, C.H. (2012) Artificial Neural Networks for Intelligent Manufacturing. Sprin- 
ger Science \& Business Media, Berlin.

[14] Jacques, L. and El Ouafi, A. (2018) ANN Based Predictive Modelling of Weld Shape and Dimensions in Laser Welding of Galvanized Steel in Butt Joint Configurations. Journal of Minerals and Materials Characterization and Engineering, 6, 316-332. https://doi.org/10.4236/jmmce.2018.63022

[15] Oussaid, K., El Ouafi, A. and Chebak, A. (2019) Experimental Investigation of Laser Welding Process in Overlap Joint Configuration. Journal of Materials Science and Chemical Engineering, 7, 16-31. https://doi.org/10.4236/msce.2019.73002

[16] Oussaid, K. and El Ouafi, A. (2019) A Three-Dimensional Numerical Model for Predicting the Weld Bead Geometry Characteristics in Laser Overlap Welding of Low Carbon Galvanized Steel. Journal of Applied Mathematics and Physics, 7, 2169-2186. https://doi.org/10.4236/jamp.2019.710149 